\title{
AMIDO NATIVO E MODIFICADO DE TARO (Colocasia esculenta L. Schott): CARACTERIZAÇÃO QUÍMICA, MORFOLÓGICA E PROPRIEDADES DE PASTA
}

\author{
ELISÂNDRA COSTA ALMEIDA* \\ PUSHKAR SINGH BORA** \\ NÉSTOR ANTÔNIO HEREDIA ZÁRATE***
}

\begin{abstract}
O objetivo desta pesquisa foi comparar as propriedades do amido nativo de taro (Colocasia esculenta L. Schott), clone Macaquinho, com o seu amido modificado por oxidação para utilização pela indústria alimentícia. As amostras foram avaliadas com base nas alterações referentes à sua morfologia e propriedades funcionais. Os percentuais de grupos carboxila e carbonila obtidos após modificação classificou o tratamento empregado como brando, o qual é determinante para a caracterização funcional desse amido. A microscopia revelou grânulos com formatos circulares e poliédricos, aglomerados, tendo o amido oxidado apresentado grânulos um pouco mais volumosos. O amido oxidado mostrou maior poder de intumescimento e solubilidade na temperatura prevista para gelatinização $\left(75^{\circ} \mathrm{C}\right)$, embora tenha sido superado pelo homólogo nativo em temperatura próxima a $80^{\circ} \mathrm{C}$, com valores máximos para ambos os amidos a $95^{\circ} \mathrm{C}$. O amido oxidado também apresentou maior capacidade de absorção de água e óleo, transparência e propriedades de pasta em relação ao amido nativo. A modificação conduzida evidenciou excelentes vantagens para a indústria alimentícia que necessita de produtos com propriedades específicas em temperaturas mais baixas em virtude da rapidez desejada no preparo de sopas, molhos e sobremesas, entre outros alimentos instantâneos.
\end{abstract}

* Doutora em Ciência e Tecnologia de Alimentos, Centro de Ciências Humanas, Sociais e Agrárias, Departamento de Gestão e Tecnologia Agroindustrial, Universidade Federal da Paraíba (UFPB), Bananeiras, PB, Brasil (e-mail: elisandra.quimica@gmail.com).

** Doutor e PhD em Tecnologia de Alimentos, Centro de Tecnologia, Programa de Pós-Graduação em Ciência e Tecnologia de Alimentos, UFPB, João Pessoa, PB, Brasil (e-mail: pradesh@uol.com.br).

*** Doutor e PhD em Fitotecnia, Faculdade de Ciências Agrárias, Universidade Federal da Grande Dourados (UFGD), Dourados, MS, Brasil (e-mail: nahz@terra.com.br). 


\section{INTRODUÇÃO}

Em avaliação sobre a potencialidade de plantas tropicais, o taro apresentou o maior valor potencial de produção de amido por área entre sete espécies pesquisadas. Em função do seu rendimento agrícola evidenciou grande potencial para produção industrial de fécula (LEONEL e CEREDA, 2002), além de apresentar excelente percentual de amido em sua constituição, cerca de 80\% (DAIUTO e CEREDA, 2003).

O amido, importante fonte energética para a alimentação humana (LEONEL e CEREDA, 2002), pode ser extraído e utilizado para diversos fins (MATSUGUMA, 2006). O produto amiláceo extraído de partes comestíveis dos vegetais representa a fonte mais importante de carboidratos na alimentação humana, alcançando 80 a $90 \%$ de todos os polissacarídeos da dieta. Disponível em quantidade suficiente, o amido pode ser extraído com elevado grau pureza nos processos industriais. Formado por polímeros de glicose, o amido é depositado sob a forma de grânulos nas células que podem apresentar morfologia variada dependendo da fonte botânica (WÜRZBURG, 1989).

As principais fontes comerciais de amido no mundo são os órgãos de reserva de algumas plantas, dentre os quais os grãos de cereais (milho e arroz), algumas raízes (mandioca), tubérculos (batata) e sementes de leguminosas (feijões e ervilhas). A maior parte dos amidos dessas plantas são submetidos a diversos tipos de modificações existentes, como a oxidação. $\mathrm{O}$ amido modificado pode, em maior ou menor grau, apresentar várias propriedades que permitem usos específicos na indústria de alimentos. Há necessidade de intensificação das pesquisas sobre fontes alternativas de amido, tornando possível avaliar a qualidade de seu amido nativo e realizar modificações que possam melhorar suas qualidades funcionais, aumentando as possibilidades produtivas das indústrias alimentícias (PEREIRA, 2008; CEREDA, VILPOUX e DEMIATE, 2003).

Quando novos produtos são desenvolvidos, amidos com propriedades específicas são utilizados para conferir a funcionalidade desejada ao alimento. No entanto, essas aplicações apresentam limitações que dificultam sua utilização na forma nativa em determinados produtos. Essas limitações podem ser solucionadas com modificações pelas vias química, física, enzimática e combinação de diferentes vias, conferindo ao amido propriedades funcionais peculiares. Portanto, as aplicações de amidos dependem de suas propriedades físicas e químicas, as quais variam de uma espécie para outra, ou são influenciadas por fatores ecológicos e agronômicos (ADEBOWALE et al., 2005). Essas modificações permitem "moldar" o amido de acordo com a finalidade desejada, gerando produtos amiláceos mais específicos (ALEXANDRINO, 2006). A funcionalidade dos amidos influencia propriedades como, textura, volume, consistência, umidade e vida-de-prateleira dos alimentos (RAEKER et al., 1998).

A modificação por oxidação ocorre pela reação do amido com quantidade específica de reagente em $\mathrm{pH}$ e temperatura controlados. As diferenças nas propriedades físico-químicas dos amidos oxidados foram anteriormente atribuídas a diferenças na morfologia dos grânulos, assim como aos grupos introduzidos na estrutura molecular (KUAKPETOON, WANG e WANG, 2001). O uso de amidos oxidados pela indústria alimentícia tem aumentado devido à sua baixa viscosidade, alta estabilidade, claridade, formação de filme e propriedade de retenção de água. Assim, os amidos têm sido empregados como coberturas de alimentos, agentes ligantes em produtos de confeitaria e como emulsificantes.

A possibilidade de introduzir novas matérias-primas agrícolas como fontes de amido com características interessantes comercialmente vem suscitando o interesse dos industriais da área, visto que no Brasil existe grande variedade de fontes amiláceas ainda pouco exploradas (VILPOUX, 2003), como é o caso do taro.

Para incrementar as possibilidades de uso e atender à demanda de características reológicas específicas de novos produtos alimentícios modificou-se, quimicamente, o amido nativo de taro, clone Macaquinho por oxidação, visando a melhoria de suas propriedades funcionais. 


\section{MATERIAL E MÉTODOS}

\subsection{MATÉRIA-PRIMA}

Foram utilizados rizomas de taro (Colocasia esculenta L. Schott), clone Macaquinho, obtidos de plantas cultivadas na horta do Curso de Agronomia da Universidade Federal da Grande Dourados, Estado de Mato Grosso do Sul, Brasil.

\subsection{EXTRAÇÃO DO AMIDO}

Obteve-se o amido conforme metodologia descrita por Adebowale et al. (2005), com modificações. Os rizomas do taro foram limpos, lavados, descascados, cortados em cubos e lavados novamente com água corrente, sendo colocados em imersão em solução de metabissulfito de sódio a 0,2 g.100 mL $\mathrm{mL}^{-1}$ por 36 horas. O material foi triturado com nova solução de metabissulfito, utilizando-se liquidificador em velocidade baixa por 30 minutos, e peneirado em malha de $0,074 \mathrm{~mm}$ (200 mesh). Descartou-se o resíduo fibroso, sendo o líquido contendo amido filtrado e submetido decantação por 24 horas, seguido de centrifugação a 5.000 rpm por 15 minutos e de descarte do sobrenadante. Esse procedimento foi realizado duas vezes para eliminação das impurezas. $\mathrm{O}$ amido resultante foi seco em estufa com circulação de ar a $40 \pm 2^{\circ} \mathrm{C}$ por 24 horas, seguido de pulverização e nova fase de peneiramento (abertura $0,074 \mathrm{~mm}$ ) para ser submetido à modificação por oxidação.

\subsection{COMPOSIÇÃO CENTESIMAL DO AMIDO NATIVO}

O amido nativo de taro foi analisado quanto a composição centesimal de acordo com a AACC (1995), mediante determinações do conteúdo de umidade (método $n^{\circ}$ 44-15), teor de nitrogênio total pelo método de Kjeldahl $n^{\circ} 46-13$ (usando-se o fator 6,25 para obter o teor de proteína bruta), teor de cinzas (método $\mathrm{n}^{\circ} 08-01$ ) e teor de lipídios pelo método $\mathrm{n}^{\circ}$ 30-20. $\mathrm{O}$ teor de amido foi determinado pela hidrólise ácida do amido a glicose por meio de autoclavação e caracterização da glicose pelos reagentes de Fehling A e B por titulação, utilizando-se azul de metileno com indicador (calculado em g.100 $\mathrm{g}^{-1}$ ), segundo o Instituto Adolfo Lutz (2008).

\subsection{MODIFICAÇÃO POR OXIDAÇÃO}

Modificou-se o amido do taro por tratamento oxidativo com hipoclorito de sódio ( $\mathrm{NaOCl}$ ), conforme metodologia descrita por Forssel et al. (1995). Preparou-se a pasta de amido a $50 \mathrm{~g}$. $100 \mathrm{~mL}^{-1}$, ajustando o $\mathrm{pH}$ para 9,5 com hidróxido de sódio a 2M. Em seguida, $10 \mathrm{~g}$ de hipoclorito de sódio foram adicionados à pasta durante 30 minutos, mantendo-se o pH entre 9,0 e 9,5e temperatura de $30 \pm 2^{\circ} \mathrm{C}$ sob agitação constante. Deixou-se a mistura reagir por 10 minutos e ajustou-se o pH para 7,0 com ácido sulfúrico a 1M. Recuperou-se o amido por filtração (lavando-o quatro vezes com água destilada), que foi seco em estufa com circulação de ar a $45^{\circ} \mathrm{C}$ por 24 horas. Em seguida, o amido modificado foi pulverizado e peneirado (abertura 0,074 $\mathrm{mm}$ ).

\subsection{DETERMINAÇÃO DO CONTEÚDO DE CARBOXILA DO AMIDO OXIDADO}

Determinou-se o conteúdo de carboxilas na amostra pelo método de Parovuori et al. (1995), suspendendo $5 \mathrm{~g}$ do amido oxidado em $25 \mathrm{~mL}$ de ácido clorídrico $(\mathrm{HCl})$ 0,1M por 40 minutos em temperatura ambiente sob agitação mecânica. Após a acidificação, a amostra foi recuperada e lavada exaustivamente com água destilada por meio de filtração até que o pH da água de lavagem estivesse neutro. A amostra foi transferida para erlenmeyer e dispersa em $300 \mathrm{~mL}$ de água destilada. Aqueceu-se a suspensão até ebulição com agitação contínua para promover a gelatinização do amido. Após esse período, a pasta ainda quente foi titulada com solução padrão de hidróxido de sódio $0,1 \mathrm{M}$ utilizando-se fenoftaleína como indicador. Para quantificar principalmente os ácidos 
graxos complexados com a amilose efetuou-se titulação em branco, usando $5 \mathrm{~g}$ do amido nativo. Obteve-se o conteúdo de carboxilas conforme a equação 1:

$$
\text { Carboxilas }(\mathrm{COOH})\left(\mathrm{g} \cdot 100 \mathrm{~g}^{-1}\right)=(\mathrm{Va}-\mathrm{Vb}) \cdot \mathrm{Ma} \cdot 0,045 \cdot 100 \cdot \mathrm{PA}^{-1}
$$

Em que:

$\mathrm{Va}=$ volume gasto na titulação do amido oxidado $(\mathrm{mL})$;

$\mathrm{Vb}=$ titulação do amido nativo $(\mathrm{mL})$;

Ma = molaridade do álcali;

$\mathrm{PA}=$ peso da amostra em base seca $(\mathrm{g})$.

\subsection{DETERMINAÇÃO DO CONTEÚDO DE CARBONILA DO AMIDO OXIDADO}

Determinou-se o conteúdo de carbonilas nas amostras conforme descrito por Smith (1967), mediante dispersão de $2 \mathrm{~g}$ do amido oxidado em $100 \mathrm{~mL}$ de água destilada. A suspensão, gelatinizada em banho-maria e resfriada até $40^{\circ} \mathrm{C}$, teve o $\mathrm{pH}$ ajustado para 3,2 , sendo adicionado $15 \mathrm{~mL}$ da solução de hidroxilamina. O recipiente contendo a amostra foi envolvido com papel alumínio e levado ao banho-maria a $40^{\circ} \mathrm{C}$ durante 4 horas. Determinou-se a hidroxilamina adicionada pela titulação rápida da mistura em pH 3,2 com ácido hidroclorídrico a 0,1M. Obteve-se o conteúdo de carbonilas pela equação 2 :

$$
\text { Carbonilas }(\mathrm{C}=\mathrm{O})\left(\mathrm{g} \cdot 100 \mathrm{~g}^{-1}\right)=(\mathrm{Vb}-\mathrm{Va}) \cdot \mathrm{Ma} \cdot 0,028 \cdot 100 \cdot \mathrm{PA}^{-1}
$$

Em que:

$\mathrm{Vb}=$ volume gasto na titulação do amido nativo $(\mathrm{mL})$;

$\mathrm{Va}=$ volume gasto na titulação do amido oxidado $(\mathrm{mL})$;

$\mathrm{Ma}=$ molaridade do ácido;

$\mathrm{PA}=$ peso da amostra em base seca $(\mathrm{g})$.

\subsection{CARACTERIZAÇÃO DO AMIDO NATIVO E MODIFICADO POR OXIDAÇÃO}

\subsubsection{Microscopia dos grânulos (estrutura granular)}

Aproximadamente $2 \mathrm{~g}$ por $100 \mathrm{~mL}$ dos amidos nativo e oxidado foram dispersos em álcool isopropílico e gotas dessas dispersões foram colocadas sobre fita adesiva à base de suporte metálico. Após a evaporação do solvente, o material seco foi recoberto com ouro (sputtering) e as amostras analisadas em microscópio eletrônico de varredura (MEV) digital, modelo LEO-1430, conforme metodologia descrita por Freitas e Tavares (2005).

\subsection{PROPRIEDADES FUNCIONAIS DOS AMIDOS NATIVO E MODIFICADO POR OXIDAÇÃO}

\subsubsection{Poder de intumescimento (inchamento) e solubilidade}

O poder de inchamento e a solubilidade dos amidos foram determinados de acordo com o método descrito por Leach, Mccowen e Schoch (1959). Pesou-se 0,1 g de amido em tubos de centrífuga, previamente pesados, adicionando-se $10 \mathrm{~mL}$ de água destilada. A suspensão, agitada em agitador de tubos por 30 segundos, foi levada ao banho-maria por 30 minutos com a temperatura variando entre 55 e $95^{\circ} \mathrm{C}$. Em seguida, os tubos foram retirados do banho, fechados e centrifugados por 15 minutos a $3.400 \mathrm{rpm}$. Alíquota de $5 \mathrm{~mL}$ foi retirada do sobrenadante e colocada em tubos de ensaio previamente pesados que foram levados à estufa a $105^{\circ} \mathrm{C}$ por 24 horas para determinação do peso do amido solubilizado (poder de solubilidade). Os tubos contendo o amido sedimentado 
foram cuidadosamente pesados e o poder de inchamento calculado conforme as equações 3 e 4 :

$$
\text { Poder de inchamento }\left(\mathrm{g} \cdot \mathrm{g}^{-1}\right)=(\mathrm{Pc}-\mathrm{Pa}) \cdot \mathrm{PA}^{-1}
$$

Em que:

$\mathrm{Pc}=$ peso do tubo com resíduo após centrifugação;

$\mathrm{Pa}=$ peso do tubo com amostra em base seca;

$\mathrm{PA}=$ peso da amostra $(\mathrm{g})$.

$$
\text { Solubilidade }\left(\mathrm{g} \cdot 100 \mathrm{~g}^{-1}\right)=(\mathrm{Pe}-\mathrm{Pt}) \cdot 100 . \mathrm{PA}^{-1}
$$

Em que:

$\mathrm{Pe}=$ peso do tubo com resíduo após evaporação;

$\mathrm{Pt}=$ peso do tubo;

$\mathrm{PA}=$ peso da amostra (g).

\subsubsection{Capacidade de absorção em água (CAA) e óleo (CAO)}

A capacidade de absorção de água e óleo foi determinada de acordo com o método descrito por Beuchat (1977). A um grama da amostra foram adicionados $10 \mathrm{~mL}$ da água destilada ou do óleo (óleo de soja Soya, Bunge - Indústria Brasileira) em tubos de centrífuga previamente secos em estufa e pesados. A suspensão foi homogeneizada durante 30 segundos e deixada em repouso por 30 minutos. Os tubos foram fechados e centrifugados por 15 minutos a $3400 \mathrm{rpm}$. Descartou-se o sobrenadante e os tubos foram invertidos para escoar a água por 10 minutos. As paredes externas dos tubos foram secas e os tubos pesados. Para determinar a absorção de água ou óleo, a massa da água ou do óleo absorvidos foi expressa em g.100 g $\mathrm{g}^{-1}$ de amido em base seca, conforme cálculo realizado com base na equação 5 :

$$
\text { CAA ou CAO }\left(\mathrm{g} \cdot 100 \mathrm{~g}^{-1}\right)=(\mathrm{Pc}-\mathrm{Pt}) \cdot 100
$$

Em que:

Pc = peso do tubo com amostra após centrifugação;

$\mathrm{Pa}=$ peso do tubo com amostra em base seca.

\subsubsection{Claridade da pasta}

Determinou-se a claridade da pasta por transmitância (\%T), conforme descrito por Craig et al. (1989). Suspensões de amido (1 g.100 mL) em $10 \mathrm{~mL}$ de água foram aquecidas durante 30 minutos em banho com água fervente e agitação de 30 segundos a cada 5 minutos, sendo então agitadas e resfriadas à temperatura ambiente. A transmitância foi determinada a $650 \mathrm{~nm}$ utilizandose espectrofotômetro (marca Coleman 33D).

\subsubsection{Propriedades de pasta}

As características viscoamilográficas das amostras foram determinadas em analisador rápido de viscosidade (RVA - Rapid Visco Analyser), série 4, utilizando-se o programa Thermocline for Windows versão 2.3, perfil Standart, segundo a metodologia n'162 proposta pela International Association for Cereal Science and Technology (1995).

Para a realização da análise iniciou-se o aparelho com rotação de 960 rpm até o tempo 00:00:10 e durante o restante do teste usou-se rotação de $160 \mathrm{rpm}$. Do tempo 00:00:00 a 00:01:00, a temperatura foi de $50^{\circ} \mathrm{C}$, aos 00:04:45 chegou a $95^{\circ} \mathrm{C}$, permanecendo constante até 00:07:15 e decrescendo até $50^{\circ} \mathrm{C}$ aos 00:13:00 no final da análise. Foram avaliados os seguintes parâmetros: 
pico de viscosidade (corresponde à viscosidade máxima na curva viscoamilográfica), quebra da viscosidade (viscosidade máxima - a viscosidade mínima), viscosidade final (valor da viscosidade sob temperatura de $50^{\circ} \mathrm{C}$ no ciclo de resfriamento), retrogradação (viscosidade final - a viscosidade mínima) e temperatura de pasta (temperatura inicial de gelatinização, quando se inicia a curva da viscosidade).

A concentração de sólidos de cada amostra foi corrigida com base nos valores de porcentagem de umidade, utilizando-se a relação de $3,5 \mathrm{~g}$ da amostra para $14 \mathrm{~g} .100 \mathrm{~g}^{-1}$ de umidade em $25 \mathrm{~mL}$ de água. Os resultados foram expressos em cP ou mPa s.

\subsection{ANÁLISE ESTATÍSTICA}

Os dados da composição centesimal e das propriedades funcionais foram submetidos à estatística descritiva, com observação das médias e desvio padrão de três repetições. Para a comparação entre as médias obtidas utilizou-se a análise de variância (ANOVA) e aplicou-se o teste de Tukey ao nível de $5 \%$ de significância. As análises foram realizadas utilizando-se o programa estatístico SPSS Statistics 19.0 (SPSS, 2010).

\section{RESULTADOS E DISCUSSÃO}

\subsection{COMPOSIÇÃO CENTESIMAL DO AMIDO NATIVO}

Os resultados obtidos para a composição centesimal do amido nativo de taro clone Macaquinho, referentes aos percentuais de amido, teor de água, resíduo mineral fixo, proteínas, lipídeos e outros componentes (por diferença) estão dispostos na Tabela 1.

\section{TABELA 1 - COMPOSIÇÃO CENTESIMAL DE AMIDO NATIVO DE TARO (Colocasia esculenta L. SCHOTT) CLONE MACAQUINHO}

\begin{tabular}{lc}
\hline \multicolumn{1}{c}{ Constituintes } & Resultados $\left(\mathbf{g} \cdot \mathbf{1 0 0} \mathbf{~ g}^{-1}\right)^{*}$ \\
\hline Amido & $74,65 \pm 0,07$ \\
Umidade & $11,43 \pm 0,21$ \\
Resíduo mineral & $0,35 \pm 0,25$ \\
Proteínas & $2,36 \pm 0,01$ \\
Lipídeos & $1,25 \pm 0,12$ \\
Fibras & $1,22 \pm 0,23$ \\
Outros componentes (por diferença) & 8,74 \\
\hline
\end{tabular}

*Valores médios (base seca) obtidos em triplicata seguidos de desvio padrão.

No presente estudo, o amido nativo mostrou percentual amiláceo considerável $\left(74,65 \mathrm{~g} .100 \mathrm{~g}^{-1}\right)$. Alexandrino (2006), ao pesquisar o conteúdo de amido em fécula de mandioca, milho e batata, obteve resultados similares ao encontrado neste estudo. No entanto, outros pesquisadores relataram valores superiores desse constituinte em culturas semelhantes, como outras variedades de inhame, sendo 83,06, 80,98, 86,50 e 80,47 g.100 g ${ }^{-1}$, Daiuto e Cereda (2004), Brasileiro (2006), Adebowale et al. (2009) e Ojinnaka e Acobundo e Iwe (2009), respectivamente. Esse fato pode ser devido às dificuldades de extração do amido de taro em razão da possível presença de mucopolissacarídeos, que podem prejudicar a seleção desse amido durante o processo de extração e decantação. A presença desses compostos, no entanto, pode ser considerada diferencial importante em relação a outras culturas, visto que apresentam excelente propriedade como espessante (DAIUTO e CEREDA, 2004). 
A umidade do amido nativo (11,43 g.100 g $\left.{ }^{-1}\right)$ obtida encontra-se de acordo com a Instrução Normativa n. 23, de 14 de dezembro de 2005, do Ministério da Agricultura, Pecuária e Abastecimento (BRASIL, 2005a). Valores de umidade são aceitáveis para amidos em percentual menor que 14 g.100 g ${ }^{-1}$. Deve-se lembrar que o percentual de umidade em amidos é extremamente importante para controlar seu processo de secagem, além de representar parâmetro indispensável para que todos os resultados de análises subsequentes possam ser expressos em base seca.

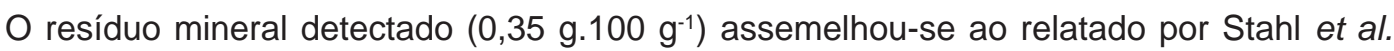
(2007) e Sá (2007) estudando amidos de pinhão e fruta-pão (0,32 g.100 g-1); Lawal (2004) e Lawal e Adebowale (2005) avaliando amidos de milho hibrido e semente de jaca $\left(0,33 \mathrm{~g} .100 \mathrm{~g}^{-1}\right)$. Tais valores enquadram-se nos padrões da RDC $n^{\circ} 263$, de 22 de setembro de 2005, da ANVISA que permite valores para cinzas de até $0,50 \mathrm{~g}^{1} 100 \mathrm{~g}^{-1}$ (BRASIL, 2005b).

Obteve-se teor de proteínas (2,36 g.100 g-1) elevado para amidos em geral, tendo em vista que esse componente é considerado como impureza. Contudo, Maieves (2010) em pesquisa realizada com raízes de mandioca detectou percentuais de proteínas que variaram de 2,22 a 3,66 g.100 g-1.

O percentual lipídico do amido (1,25 g.100 g-1) também foi considerado um pouco elevado, pois se trata de componente interferente no amido. Uma das suas principais características é a formação de complexo com a amilose, o que pode comprometer algumas das propriedades do amido. No entanto, foram encontrados na literatura valores de $1,52 \mathrm{~g} .100 \mathrm{~g}^{-1} \mathrm{em}$ amido de milho relatados por Alexandrino (2006) e de $1,20 \mathrm{~g} .100 \mathrm{~g} \mathrm{~g}^{-1} \mathrm{em}$ amidos de trigo e milho citados por Batista, Silva e Liberato (2010)

\subsection{CONTEÚDO DE CARBOXILA E CARBONILA DO AMIDO OXIDADO}

Os percentuais de grupos carboxílicos e carbonílicos obtidos para amido modificado de taro pelo processo oxidativo estão dispostos na Tabela 2, não sendo encontrados na literatura resultados de outros autores para esses parâmetros em taro.

\section{TABELA 2 - CONTEÚDO DE CARBOXILA E CARBONILA DO AMIDO OXIDADO DE TARO (Colocasia esculenta L. Schott) CLONE MACAQUINHO, COMPARADOS COM AMIDOS OXIDADOS EM OUTRAS PESQUISAS}

\begin{tabular}{lccc}
\hline \multirow{2}{*}{ Amido Oxidado } & \multicolumn{2}{c}{ Grupos $\left(\mathbf{g} \cdot \mathbf{1 0 0} \mathbf{~ g}^{-1}\right)$} & \\
\cline { 2 - 3 } Taro & Carboxila & Carbonila & Autor(es) \\
Mandioca & 0,098 & 0,11 & - \\
Mandioquinha-salsa & $0,18-0,34$ & - & Tran, Piyachomkwan e Sriroth (2007) \\
Batata & 0,13 & - & Matsuguma (2006) \\
Batata-doce & 0,35 & - & Silva et al. (2008) \\
Milho & 0,17 & - & Takizawa et al. (2004) \\
Pinhão & $0,08-1,43$ & $0,097-0,54$ & Shirai et al. (2007) \\
Semente de Jaca & 0,39 & 0,21 & Conto et al. (2011) \\
Amêndoa de Manga & 0,09 & 0,05 & Lawal e Adebowale (2005) \\
\hline
\end{tabular}


O amido de taro oxidado apresentou percentuais aproximados de carboxila $\left(0,098 \mathrm{~g} \cdot 100 \mathrm{~g}^{-1}\right)$ e carbonila $\left(0,11 \mathrm{~g} \cdot 100 \mathrm{~g}^{-1}\right)$, isto ocorre porque durante a reação os grupos hidroxilas nas moléculas de amido são oxidados a grupos carbonilas e depois a carboxilas (WÜRZBURG,1986), demonstrando a eficiência do processo de modificação conduzido. Valores aproximados para esses parâmetros foram verificados por Conto et al. (2011) que quantificaram o percentual dos grupos carboxila e carbonila em amido de pinhão com diferentes concentrações de agente oxidante. Obtiveram resultado de 0,08 g.100 g g-1 de carboxila e 0,097 g.100 g-1 de carbonila para a menor concentração aplicada.

Mendes (2011), pesquisando amido oxidado de amêndoa de manga, apresentou resultado coerente para percentual de carboxila $\left(0,09 \mathrm{~g} .100 \mathrm{~g}^{-1}\right)$ e Lawal e Adebowale (2005), estudando amido de semente de jaca oxidado, encontraram valor aproximado para o percentual de carbonila $\left(0,21 \mathrm{~g} \cdot 100 \mathrm{~g}^{-1}\right)$ em relação ao amido de taro modificado por oxidação.

Pode-se verificar que os resultados obtidos de outras fontes de amido oxidadas são muito variáveis, provavelmente porque o tipo e a quantidade desses grupos funcionais formados nas moléculas de amido durante a oxidação dependerá do tempo de reação, da temperatura e do $\mathrm{pH}$ empregados (SANGSEETHONG, LERTPHANICH e SRIROTH, 2009). Elevados percentuais de grupos carboxílicos e carbonílicos podem ser devidos à severidade do processo oxidativo empregado na modificação. Os resultados obtidos neste estudo para amido oxidado de taro denotam tratamento brando, quando comparados com outras pesquisas.

\subsection{CARACTERIZAÇÃO DOS AMIDOS NATIVO E MODIFICADO POR OXIDAÇÃO}

\subsubsection{Microscopia dos grânulos (estrutura granular)}

As micrografias dos grânulos de amido nativo e oxidado do taro Macaquinho, obtidas por microscopia eletrônica de varredura (MEV) estão expostas na Figura 1.
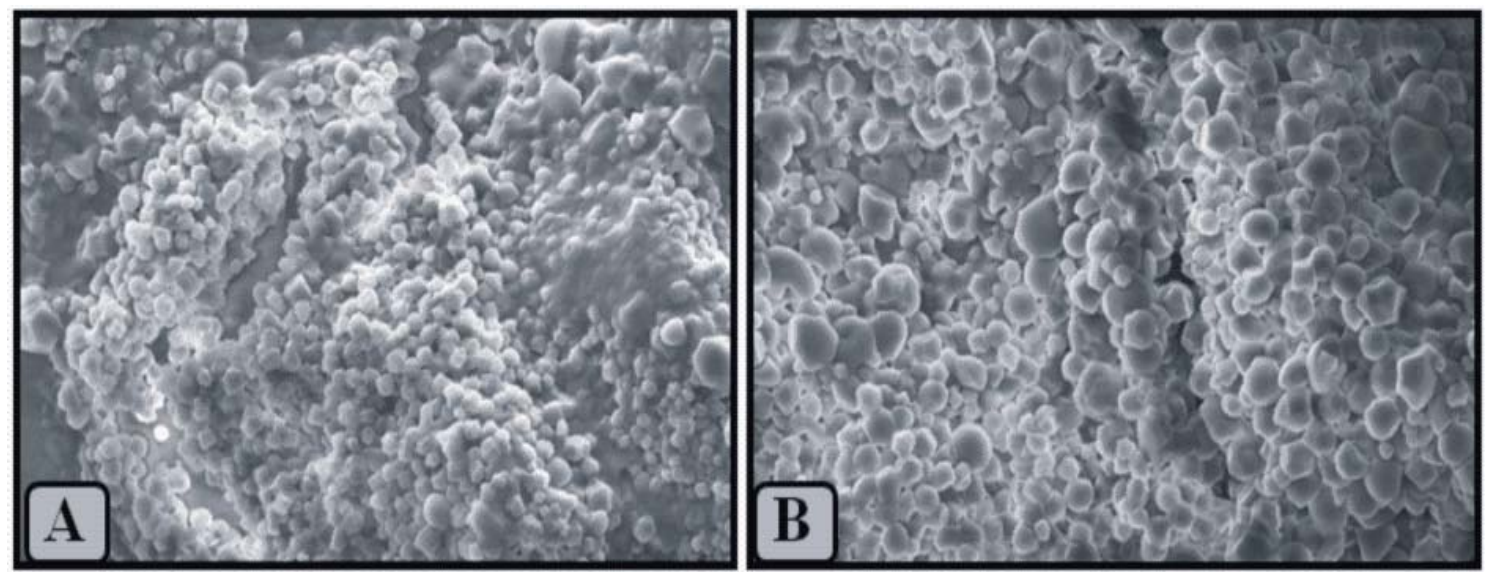
FIGURA 1 - MICROGRAFIAS DOS GRÂNULOS DE AMIDO DE TARO (Colocasia esculenta L. Schott) CLONE MACAQUINHO NATIVO (A) E MODIFICADO POR OXIDAÇÃO (B), OBSERVADOS EM MICROSCÓPIO ELETRÔNICO DE VARREDURA (MEV) (APROXIMAÇÃO DE 5000X)

Verificou-se pela análise das micrografias que o amido oxidado mostrou características morfológicas semelhantes ao amido nativo, quando observados em MEV. O amido nativo apresentava grânulos com formatos circulares e poliédricos bastante aglomerados. Leonel (2007) analisando grânulos de amidos de diferentes fontes botânicas observou em amido de taioba grânulos com formas circulares e poliédricas, semelhantes ao obtido em amidos de taro pesquisados neste estudo. 
Depois de modificados os grânulos dos amidos oxidado não sofreram alterações muito significativas em suas morfologias, porém apresentaram dimensões singularmente diferentes. Gonzalez e Perez (2002), pesquisando amidos modificados também verificaram alteração na estrutura granular após a modificação.

O amido oxidado apresentou grânulos um pouco mais inchados e também bastante aglomerados. Esses resultados estão correlacionados com o maior poder de intumescimento dos amidos com esse tipo de modificação devido à introdução dos grupos carboxila no seu interior, favorecendo a absorção de água na região amorfa e aumentando o volume dos grânulos. Esses efeitos também foram observados por Zhang, Chen e Zhang (2011) analisando amidos modificados de diversas fontes e por Conto et al. (2011), analisando amido de pinhão modificado por oxidação.

Deve-se salientar que todas as características morfológicas observadas em amido de taro nativo e oxidado são extremamente importantes na determinação das propriedades funcionais desses amidos.

\subsection{PROPRIEDADES FUNCIONAIS DOS AMIDOS NATIVO E MODIFICADO POR OXIDAÇÃO}

\subsubsection{Poder de intumescimento (inchamento) e solubilidade}

Os resultados obtidos para o poder de intumescimento (inchamento) dos amidos de taro Macaquinho nativo e oxidado, em função da temperatura, estão expostos na Figura 2.

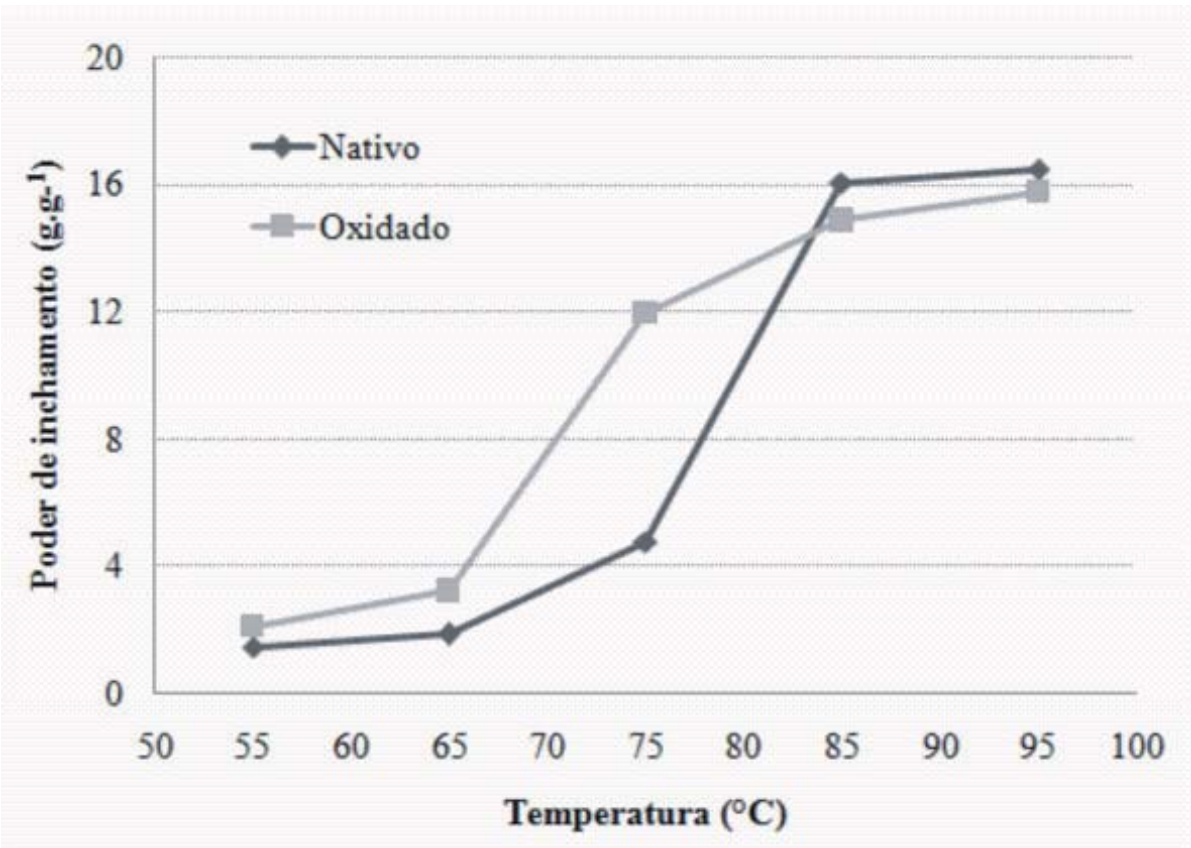

\section{FIGURA 2 - EFEITO DA VARIAÇÃO DE TEMPERATURA NO PODER DE INTUMESCIMENTO (INCHAMENTO) DO AMIDO DE TARO (Colocasia esculenta $L$. Schott) CLONE MACAQUINHO NATIVO E MODIFICADO POR OXIDAÇÃO}

Os maiores valores encontrados para o poder de intumescimento $(\mathrm{PI})$ foram observadas a partir de $75^{\circ} \mathrm{C}$, ou seja, acima da temperatura prevista para gelatinização. $\mathrm{O}$ amido oxidado mostrou poder de intumescimento bem maior a essa temperatura em comparação com seu homólogo nativo, o que é bastante favorável. A indústria alimentícia necessita cada vez mais de produtos que apresentem propriedades alteradas em temperaturas cada vez mais baixas em virtude da rapidez 
desejada no preparo de produtos como, sopas, molhos e sobremesas, entre outros alimentos instantâneos.

Ambos os amidos obtiveram poder de intumescimento máximo a $95^{\circ} \mathrm{C}$, temperatura máxima avaliada, com valores aproximados (16,49 e 15,74 g. $\mathrm{g}^{-1}$, respectivamente). Takizawa et al. (2004) ao pesquisarem amidos de diferentes fontes botânicas obtiveram 15,30 g.g $\mathrm{g}^{-1}$ para o poder de intumescimento de amido de milho nativo a $90^{\circ} \mathrm{C}$. Resultados aproximados para amido oxidado foram reportados por Takizawa et al. (2004), estudando amido de cenoura peruana $\left(15,90 \mathrm{~g} \cdot \mathrm{g}^{-1}\right)$.

Os resultados obtidos para solubilidade em amidos de taro nativo e oxidado, variando conforme a temperatura, estão expostos na Figura 3.

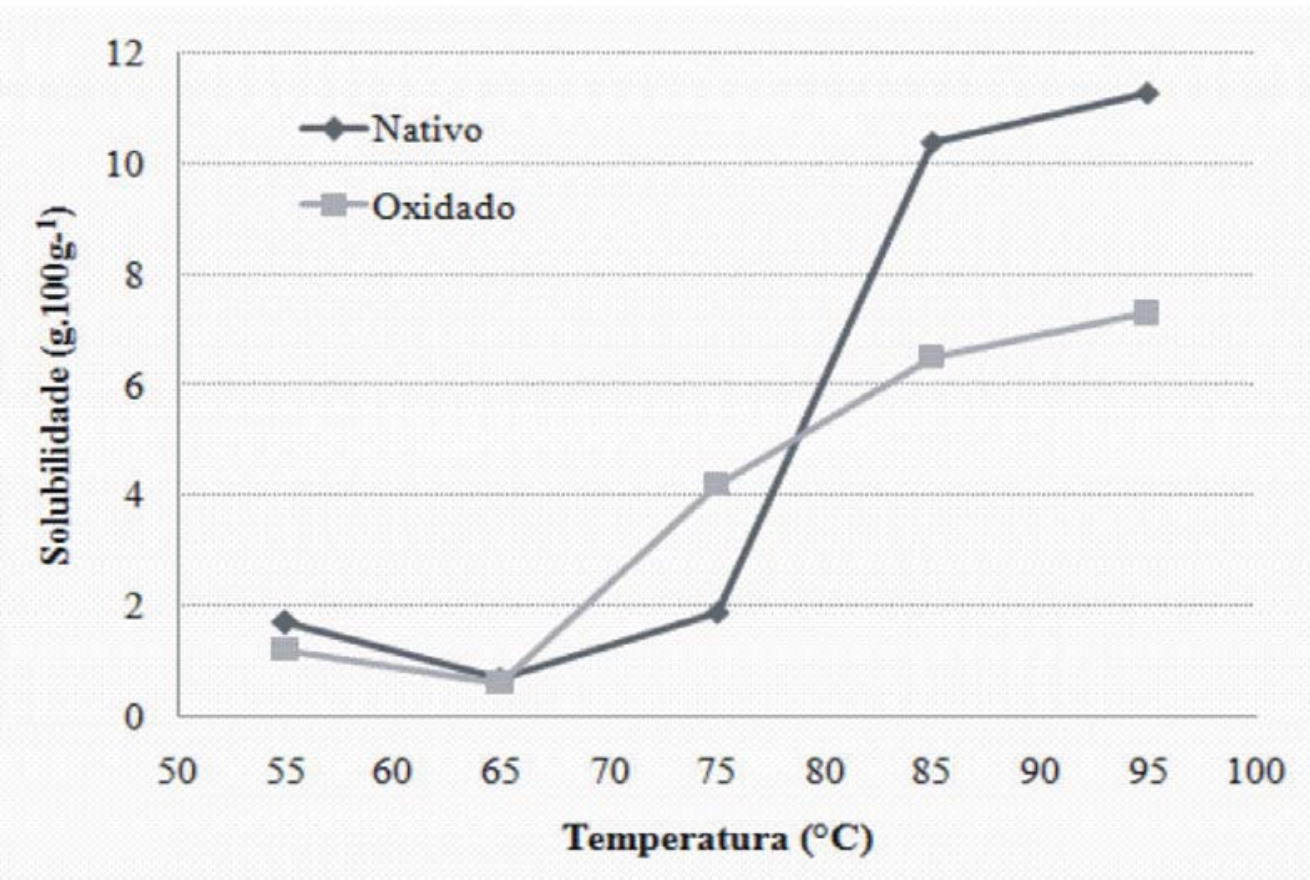

\section{FIGURA 3 - EFEITO DA VARIAÇÃO DE TEMPERATURA NA SOLUBILIDADE DO AMIDO DE TARO (Colocasia esculenta L. Schott) CLONE MACAQUINHO NATIVO E MODIFICADO POR OXIDAÇÃO}

Da mesma forma que o poder de intumescimento, os maiores resultados encontrados para solubilidade foram constatados a partir de $75^{\circ} \mathrm{C}$ (acima da temperatura prevista para gelatinização). Os amidos de taro nativo e oxidado tiveram seu percentual de solubilidade aumentado com a elevação da temperatura após alcançarem $65^{\circ} \mathrm{C}$, quando se verificou maior solubilidade na mais alta temperatura pesquisada $\left(95^{\circ} \mathrm{C}\right)$, sendo $11,30 \mathrm{~g} .100 \mathrm{~g}^{-1}$ para o amido nativo e $7,33 \mathrm{~g} .100 \mathrm{~g}^{-1}$ para o amido oxidado.

Takizawa et al. (2004) ao analisarem os amidos de batata-doce a $90^{\circ} \mathrm{C}$ obtiveram dados aproximados (10,0 g. $\left.100 \mathrm{~g}^{-1}\right)$ aos observados neste estudo. Também encontraram resultados de solubilidade semelhantes para amido oxidado de milho ceroso $\left(8,0 \mathrm{~g} .100 \mathrm{~g}^{-1}\right)$ em temperatura inferior a $60^{\circ} \mathrm{C}$. Verificaram que amidos oxidados apresentavam elevada claridade de pasta ou maior transparência, o que pode resultar em diminuição da solubilidade.

\subsubsection{Capacidade de absorção de água (CAA) e óleo (CAO)}

Os resultados verificados para capacidade de absorção de água (CAA) e óleo (CAO) dos amidos de taro nativo e oxidado estão expostos na Figura 4. 


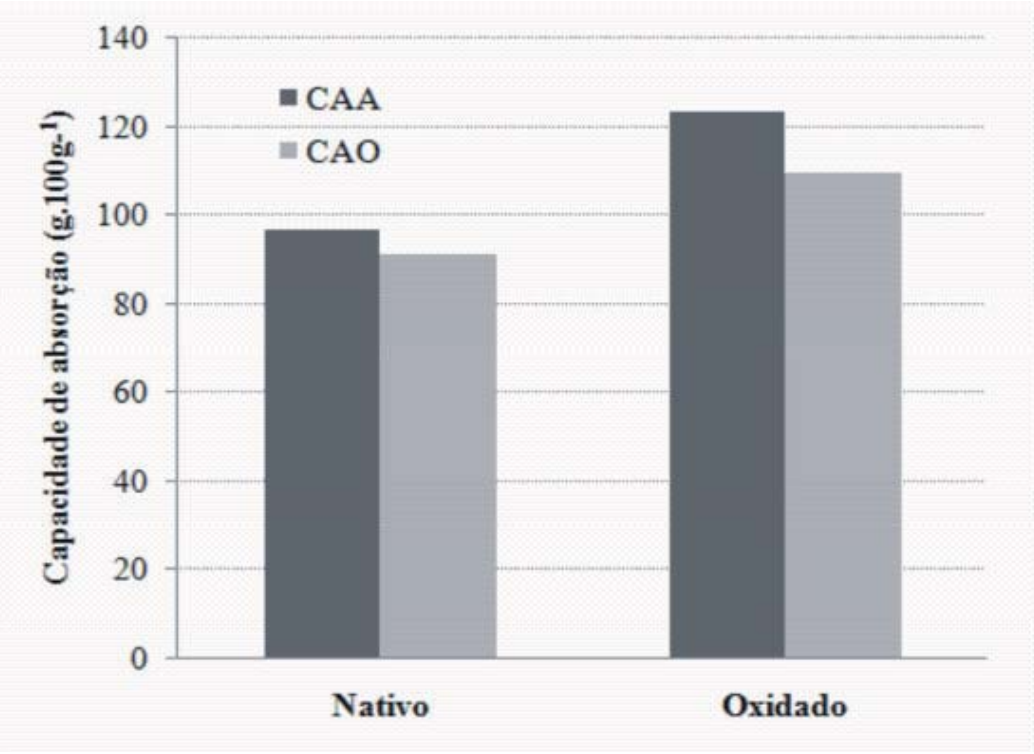

\section{FIGURA 4 - CAPACIDADE DE ABSORÇÃO DE ÁGUA (CAA) E DE ÓLEO (CAO) DO AMIDO DE TARO (Colocasia esculenta L. Schott) CLONE MACAQUINHO NATIVO E MODIFICADO POR OXIDAÇÃO}

Observou-se maior hidroficilidade para o amido submetido a oxidação (Figura 4), com

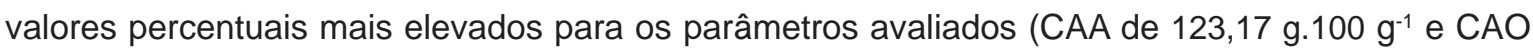
de 109,45 g.100 g-1). Já o amido nativo obteve CAA de 96,80 g.100 g-1 e CAO de 91,26 g.100 g ${ }^{-1}$. A melhoria na capacidade de absorção de água e de óleo do amido oxidado pode resultar da introdução de grupos funcionais, carboxila e carbonila na molécula de amido (RIBEIRO, 2006). Esses grupos, não sendo muito volumosos, podem ter restringido o acesso à água e elevado um pouco o percentual de absorção de seu homólogo nativo. Resultados similares foram reportados por Mendes (2011) que obteve CAA de 98,58 g.100 g-1 para amido nativo de amêndoa de semente de manga. Ribeiro (2006), avaliando amido oxidado de mandioca percebeu que a tendência hidrofóbica aumentou após o tratamento oxidativo em relação ao amido nativo. Esse comportamento também foi observado por Lawal (2004) e Adebowale, Afolabi e Lawal (2002).

\subsubsection{Claridade da pasta}

Os resultados obtidos para claridade da pasta dos amidos de taro Macaquinho nativo e oxidado estão expostos na Figura 5.

Os amidos nativo e oxidado mostraram no primeiro dia percentual de transmitância mais baixos (7,00 e 6,90\%, respectivamente) que se mantiveram estáveis apenas até o terceiro dia. A partir de então, os dois amidos mostraram tendência à retrogradação diminuída, gradualmente, sendo mais pronunciada no amido oxidado. Sá (2007) pesquisando amido de fruta-pão observou alteração na claridade da pasta com os dias de estocagem, principalmente nas primeiras 48 horas, assim como Singh, McCarthy e Singh (2006). Sangssethong, Lertphanich e Sriroth (2009) estudando amido de mandioca oxidado perceberam que a presença do grupo funcional hidrofílico, como grupos carboxila, pode ser responsável pela maior transparência de pasta em função da correlação positiva entre o conteúdo carboxílico e a transmissão da luz em amidos oxidados. Miles et al. (1985) sugeriram que o desenvolvimento precoce de retrogradação do amido é dominado pela associação de amilose. Portanto, é possível que durante o processo oxidativo o amido tenha mais ou menos partes dos grupos carboxílicos ligados às moléculas de amilose, retardando assim a sua reassociação. Esse fato também foi constatado por Song et al. (2010) em amido oxidado de milho. 


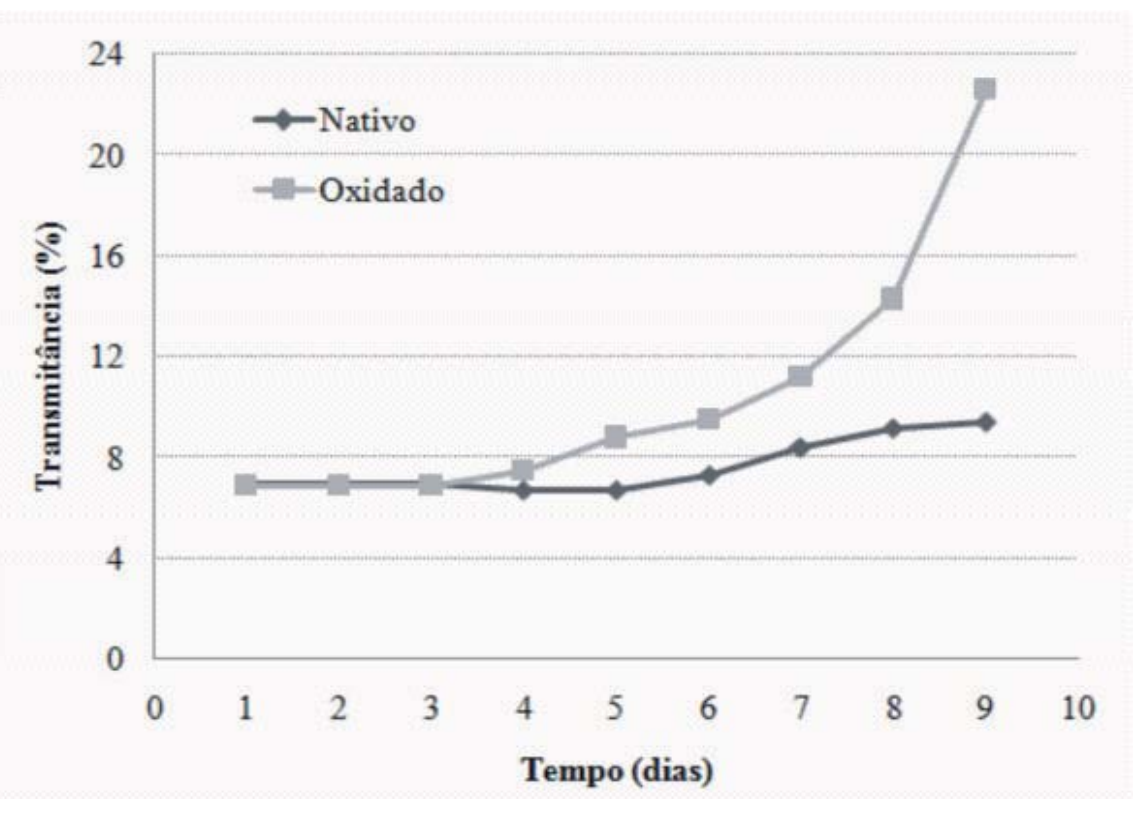

FIGURA 5 - CLARIDADE DA PASTA (\% TRANSMITÂNCIA) DO AMIDO DE TARO (Colocasia
esculenta L. Schott) CLONE MACAQUINHO NATIVO E MODIFICADO POR OXIDAÇÃO

3.4.4 Propriedades de pasta

Os resultados referentes às propriedades de pasta, avaliadas pelo RVA, dos amidos de taro Macaquinho nativo e oxidado, caracterizando os perfis viscoamilográficos desses amidos estão expostos na Tabela 3.

\section{TABELA 3 - PROPRIEDADES DE PASTA POR RVA DOS AMIDOS NATIVO E MODIFICADO POR OXIDAÇÃO DE TARO (Colocasia esculenta L. Schott) CLONE MACAQUINHO}

\begin{tabular}{|c|c|c|c|c|c|}
\hline \multirow{2}{*}{ Amidos } & \multicolumn{4}{|c|}{ Viscosidade em cP ou mPa s } & \multirow{2}{*}{$\begin{array}{c}\text { Temperatura } \\
\text { de pasta } \\
\left({ }^{\circ} \mathrm{C}\right)\end{array}$} \\
\hline & $\begin{array}{c}\text { Pico de } \\
\text { viscosidade }\end{array}$ & $\begin{array}{c}\text { Quebra da } \\
\text { viscosidade }\end{array}$ & Viscosidade final & Retrogradação & \\
\hline Nativo & $2141 \pm 107,48^{a}$ & $1025,5 \pm 6,36^{a}$ & $1638 \pm 106,07^{a}$ & $521 \pm 2,83^{a}$ & $78,45 \pm 0,07^{a}$ \\
\hline Oxidado & $3142 \pm 62,23^{b}$ & $1436 \pm 14,14^{b}$ & $4300 \pm 25,46^{b}$ & $2594 \pm 50,91^{b}$ & $77,55 \pm 0,50^{\mathrm{ab}}$ \\
\hline
\end{tabular}

Valores médios obtidos em triplicata seguidos de desvio padrão.

Letras minúsculas diferentes dispostas verticalmente diferem significativamente $(p<0,05)$ aplicando o teste de Tukey, usando a análise de variância ANOVA.

Observou-se que o maior pico de viscosidade foi obtido pelo amido oxidado, com $3142 \mathrm{cP}$, seguido pelo amido nativo, com $2141 \mathrm{cP}$. Isto ocorre porque durante a fase inicial de aquecimento, o aumento na viscosidade provocado pelo inchamento dos grânulos é registrado.

Gunaratne e Corke (2007) pesquisando amidos nativos de alguns rizomas constataram picos de viscosidade aproximados aos reportados neste estudo, sendo 159 RVU (ou $1844 \mathrm{CP}$ ) e 165 RVU (ou 1914 cP) em amidos de inhame e taro, respectivamente. Yanemoto, Calori-Domingues e Franco (2007), estudando amidos nativos de várias espécies de trigo constataram valor máximo de 194 RVU (ou 2250 cP). Espinosa-Solis, Jane e Bello-Perez (2009) obtiveram 191,1 RVU 
(ou 2216,5 cP) analisando amido nativo de polpa de manga.

No caso do amido modificado oxidado houve aumento na capacidade de inchamento devido aos respectivos grupos funcionais que penetram mais no grânulo, provocando maiores picos de viscosidade. Segundo Thomas e Atwell (1999), polímeros com menor massa incham mais rapidamente (como o grupo carbonila e carboxila) e começam a ser lixiviados dos grânulos. Assim o pico de viscosidade é obtido quando a quase totalidade dos grânulos estão inchados e não ocorreu ainda o alinhamento das moléculas dos polímeros solubilizados (caracterizado pela quebra da viscosidade). Mendes (2011) pesquisando amidos modificados de amêndoa de semente de manga obteve viscosidade máxima de $3071,5 \mathrm{cP}$, semelhante ao encontrado nesta pesquisa.

Ao analisar a tendência para retrogradação verificou-se que o amido oxidado apresentou maior capacidade para retrogradar (2594 cP) em relação ao amido nativo (521 cP). Tal fato pode ser explicado devido à elevada capacidade de cristalização das moléculas dos amidos oxidados, que ocorre pelo maior número de ligações de hidrogênio entre as moléculas adjacentes e a amilose (MILES et al., 1985), retardando sua reassociação. Esse fato também foi observado por Song et al. (2010) analisando amido oxidado de milho. Outros pesquisadores constataram tendência à retrogradação em amidos nativos correspondentes ao obtido neste estudo. Singh et al. (2009) obtiveram $405 \mathrm{cP}$ em variedade de amido de batata e Peroni (2003) trabalhando com amido de mandioca observou $61 \mathrm{RVU}$ (ou 707,5cP).

A menor temperatura de pasta, apesar da pouca variação, foi constatada no amido oxidado $\left(77,55^{\circ} \mathrm{C}\right)$, seguido pelo nativo $\left(78,45^{\circ} \mathrm{C}\right)$. É importante ressaltar que amidos com temperaturas de pasta mais baixas apresentam maior potencial para aplicação em alimentos, principalmente naqueles de preparo instantâneo, pois atingem as características ideais mais rapidamente.

\section{CONCLUSÃo}

Com base nos experimentos realizados constatou-se que o extrato amiláceo nativo de taro do clone pesquisado contém considerável percentual de amido e baixo teor de substâncias consideradas contaminantes, não influenciando suas propriedades funcionais.

$\mathrm{O}$ amido nativo pode ser utilizado em produtos que necessitem de partículas menores, com maior solubilidade e menor viscosidade, como sucos de frutas desidratados, refrigerantes, e outros. O amido oxidado, por apresentar partículas maiores em relação ao nativo, atrai o interesse das indústrias alimentícias devido sua influência sobre as propriedades de processamento de alimentos que necessitem de maior expansão, podendo ser aplicado em panificação e produtos cárneos embutidos, entre outros, proporcionando maior retenção da umidade e equilibrando as emulsões.

$\mathrm{O}$ amido modificado por oxidação também apresentou maior poder de intumescimento em temperaturas mais baixas $\left(75^{\circ} \mathrm{C}\right)$, maior valor para a capacidade de absorção de água em relação à absorção de óleo, podendo ser usado em produtos para fritura (oferecendo mais crocância). Devido à sua viscosidade mais elevada, resistência à agitação mecânica e menor temperatura de pasta pode ser aplicado em produtos instantâneos, como cremes e sobremesas. No entanto, sua maior tendência a retrogradar torna o amido modificado inapropriado para fórmulas infantis ou sopas desidratadas.

\section{ABSTRACT}

\section{NATIVE AND MODIFIED STARCH OF TARO (Colocasia esculenta L. Schott): CHEMICAL AND MORPHOLOGICAL CHARACTERIZATION AND PASTE PROPERTIES}

The objective of this study was to compare the properties of native starch from taro (Colocasia esculenta L. Schott), clone "Macaquinho" with its starch modified by oxidation to be utilized in the food industry. The samples were evaluated based on the alterations of morphology and functional properties. The percentage of carbonyl and carboxyl groups obtained after modification, classify the applied treatment as mild, and that is a determining 
factor for the functional characterization of starch. Microscopy revealed beads with circular and polyhedral, clusters, where the oxidized starch granules showed a little more bulky. The oxidized starch obtained the highest swelling power and solubility due to gelatinization temperature $\left(75^{\circ} \mathrm{C}\right)$, although it has been overcome by the native starch at a temperature close to $80^{\circ} \mathrm{C}$, with maximum values for both starches at $95^{\circ} \mathrm{C}$. The oxidized starch presented better capability of absorbing water and oils, transparency and paste properties in comparison with native starch. The performed modification presents great advantages for the food industry that needs more products with specific properties at lower temperatures, due to the speed required to prepare products such as soups, sauces, desserts, and other instant foods.

KEY-WORDS: TARO STARCH; MODIFIED STARCH; Colocasia esculenta; MORPHOLOGY; FUNCTIONAL PROPERTIES.

\section{REFERÊNCIAS}

1 AACC. American Association of Cereal Chemists. Approved methods of the American Association of Cereal Chemists. $7^{\text {th }}$ ed. St, Paul, Minnesota, 1995.

22 ADBOWALE, K.O.; AFOLABI, T.A.; LAWAL, O. Isolation, chemical modification and physicochemical characterization of bambarra groundnut (Voandzeia subterranean) starch and flour. Food Chem., v. 78, p. 305-311, 2002.

3 ADEBOWALE, K.O.; HENLE, T.; SCHWARZENBOLZ, U.; DOERT, T. Modification and properties of African yan bean (Sphenostylis stenocarpa Holchst. Ex A. Rich) Harms starch I: heart moisture treatments and anneling. Food Hydrocol., v. 23, p. $1947-1957,2009$

4 ADEBOWALE, K.O.; OLU-OWOLABI, B.I.; OLAWUMI, E.K.; LAWAL, O.S. Functional properties of native, physically and chemically modified breadfruit (Artocarpus artilis) starch. Ind. Cr. and Prod., v. 21, p. 343-351, 2005.

5 ALEXANDRINO, C.D. Utilização dos amidos de milho e de batata na elaboração de tapioca. 2006. 70 f. Dissertação (Mestrado em Tecnologia de Alimentos) - Universidade Federal do Ceará, Fortaleza, 2006.

6 BATISTA, W.P.; SILVA, C.E.M.; LIBERATO, M.C. Propriedades químicas e de pasta dos amidos de trigo e milho fosforilados. Ciên. e Tecnol. de Alim., v. 30, n. 1, p. 88-92, 2010.

7 BEUCHAT, L.R. Functional and electrophoretic characteristics of succinylated peanut flour protein. J. of Agric. and Food Chem., v. 25, p. 258-261, 1977.

8 BRASIL. Ministério da Agricultura, Pecuária e Abastecimento. Instrução Normativa $n^{\circ}$ 23, de 14 de dezembro de 2005. Regulamento técnico de identidade e qualidade dos produtos amiláceos derivados da raiz da mandioca. Diário Oficial [da] República Federativa do Brasil, Brasília, 15 de dezembro, 2005a, Seção 1, p. 5.

9 BRASIL. Ministério da Saúde. Agência Nacional de Vigilância Sanitária. Resolução nº 263, de 22 de setembro de 2005. Aprova o regulamento técnico para produtos de cereais, amidos, farinhas e farelos. Diário Oficial [da] República Federativa do Brasil, Brasília, 23 de setembro, 2005b, Seção 1, p. 368.

10 BRASILEIRO, O.L. Comparação das propriedades funcionais do amido de inhame (Dioscorea cayennensis) nativo e modificado por acetilação e succinilação. 2006. 88 f. Dissertação (Mestrado em Ciência e Tecnologia de Alimentos) - Universidade Federal da Paraíba, João Pessoa, 2006.

11 CEREDA, M.P.; VILPOUX, O.; DEMIATE, I.M. Amidos modificados. In: CEREDA, M.P.; VILPOUX, O.F. Tecnologia, usos e potencialidades de tuberosas amiláceas latino americanas. São Paulo: Fundação Cargill, 2003. v. 3, p. $246-332$.

12 CRAIG, S.A.S.; MANINGAT, C.C.; SEIB, P.A.; HOSENEY, R.C. Starch paste clarity. Cer. Chem., v. 66, n. 3, p. 173-182, 1989.

13 CONTO, L.C; PLATA-OVIEDO, M.S.V; STEEL, C.J.; CHANG, Y.K. Physicochemical, morphological, and pasting properties of Pine nut (Araucaria angustifolia) starch oxidized with different levels of sodium hypochlorite. Starch/Stärke, v. 63, p. 198-208, 2011.

14 DAIUTO, E. R.; CEREDA, M. P. Processos de produção de fécula de mandioca: comparação Tailândia e China. In CEREDA, M. P.; VILPOUX, O. Tecnologia, usos e potencialidades de tuberosas amiláceas latino- americanas. São Paulo: Fundação Cargill, 2004. v. 3, p. 178.

15 DAIUTO, E.R.; CEREDA, M.P. Inhame. In: CEREDA, MP. (Coord.) Tecnologia, usos e potencialidades de tuberoses amiláceas latino americanas. São Paulo: Fundação Cargill, 2003. v. 3, p. 142-154.

16 ESPINOSA-SOLIS, V.; JANE, J.; BELLO-PEREZ, AL. Physicochemical characteristics of starches from unripe fruits of mango and banana. Starch/Stärke, v. 61, p. 291-299, 2009.

17 FORSSEL, P.; HAMUNEM, A.; AUTIO, K.; SUORTTI, T.; POUTANEN, K. Hypochlorite oxidation of barley and potato starch. Starche/Stharkë, v. 47, p. 371-377, 1995. 
18 FREITAS, M.C.J.; TAVARES, D.Q. Caracterização do grânulo de amido de bananas (Musa AAA-nanicão E Musa AABterra). Ciênc. Tecnol. Aliment., v. 25, n. 2, p. 217-222, 2005.

19 GONZALEZ, Z.; PEREZ, E. Effect of acetylation on some properties of rice starch. Starch/Stärke, v. 54, p. 148-154, 2002.

20 GUNARATNE, A.; CORKE, H. Functional properties of hydroxypropylated, cross-linked, and hydroxypropylated crosslinked tuber and root starches. Cereal Chem., v. 84, n. 1, p. 30-37, 2007.

21 INSTITUTO ADOLFO LUTZ. Normas analíticas do Instituto Adolfo Lutz: métodos químicos e físicos para análises de alimentos. $3^{\mathrm{a}}$ ed. São Paulo, 2008. v. 1.

22 INTERNATIONAL ASSOCIATION FOR CEREAL SCIENCE AND TECHNOLOGY. Rapid pasting method using the newport rapid visco analyzer. Vienna, 1995. 10 p. (Standard No 162)

23 KUAKPETOON, D.; WANG, Y.J.; WANG, L. Characterization of different starches oxidized by hypochlorite. Starch/ Stärke, v. 53, p. 211-218, 2001.

24 LAWAL, O.S.; ADEBOWALE, K.O. Physicochemical characteristics and thermal properties of chemically modified jack bean (Canavalia ensiformes) starch. Carboh. Pol., v. 60, n. 3, p. 331-341, 2005.

25 LAWAL, O.S. Succinil and acetil starch derivatives of a hybrid maize: physicochemical characteristics and retrogradation properties monitored by differential scanning calorimetry. Carboh. Res., v. 339, p. 2673-2682, 2004.

26 LEACH, H.W.; McCOWEN, L.D.; SCHOCH, T.J. Structure of the starch granule. Swelling and solubility patterns of various starches. Cereal Chem., v. 36, n. 6, p. 534-544, 1959.

27 LEONEL, M. Análise da forma e tamanho de grânulos de amidos de diferentes fontes botânicas. Ciên. e Tecnol. de Alim., v. 27, n. 3, p. 579-588, 2007.

28 LEONEL, M; CEREDA, MP. Caracterização físico-química de algumas tuberosas amiláceas. Ciên. e Tecnol. de Alim., v. 22, n. 1, p. 65-69, 2002.

29 MAIEVES, H.A. Caracterização físico-química de cultivares de mandioca, obtidos por melhoramento genético no Estado de Santa Catarina. 2010. 113 f. Dissertação (Mestrado em Engenharia de Alimentos) - Universidade Federal de Santa Catarina, Florianópolis, 2010.

30 MATSUGUMA, L.S. Caracterização do amido de mandioquinha salsa (Arracacia xanthorrhiza) nativo e modificado por oxidação. 2006. 112 f. Dissertação (Mestrado em Ciência e Tecnologia de Alimentos) - Universidade Estadual de Ponta Grossa, Ponta Grossa, 2006.

31 MENDES, M.L.M. Caracterização para fins industriais dos amidos nativo e modificado extraídos de amêndoas de sementes de manga, variedade "Tommy Atkins". 2011. 131 f. Tese (Doutorado em Ciência e Tecnologia de Alimentos) - Universidade Federal da Paraíba, João Pessoa, 2011.

32 MILES, M.J; MORRIS, V.J.; ORFORD, P.D.; RING, S.G. The role of amylose and amylopectin in the gelation and retrogradation of starch. Carboh. Res., v. 135, p. 271-281, 1985.

33 OJINNAKA, M.C.; AKOBUNDU, E.N.T.; IWE, M.O. Cocoyam starch modifications effects on functional, sensory and cookies qualities. Pakistan J. of Nut., v. 8, n. 5, p. 558-567, 2009.

34 PAROVUORI, P.; HAMUNEN, A.; FORSSEL, P.; AUTIO, K.; POUTANEN, K. Oxidation of potato starch by hydrogen peroxide. Starch/Stärke, v. 47, n. 1, p. 19-23, 1995.

35 PEREIRA, L.H.G. Obtenção e estudo das propriedades fisico-quimicas de amidos de mandioca (Manihot Esculenta) oxidados e ácido-modificados. 2008. 108 f. Dissertação (Mestrado em Ciência e Tecnologia de Alimentos) - Universidade Estadual de Ponta Grossa, Ponta Grossa, 2008.

36 PERONI, F.H.G. Características estruturais e físico-químicas de amidos obtidos de diferentes fontes botânicas. 2003. 118 f. Dissertação (Mestrado em Engenharia e Ciência de alimentos) - Universidade Estadual Paulista "Julio de Mesquita Filho", São Paulo, 2003.

37 RAEKER, M.O. et al. Granule size distribution and chemical composition of starches from 12 soft wheat cultivars. Cereal Chem., v. 75, n. 5, p. 721-728, 1998

38 RIBEIRO, A.P.L. Estudo dos amidos de mandioca nativo, modificados e modificados combinados por via química para utilização na indústria alimentícia. 2011. 110 f. Tese (Doutorado em Ciência e Tecnologia de Alimentos) Universidade Federal da Paraíba, João Pessoa, 2011.

39 SÁ, F. Avaliação das propriedades funcionais e térmicas do amido da fruta-pão (Artocarpus altilis) nativo e modificado por succinilação e hidrólise ácida. 2007. 80 f. Dissertação (Mestrado em Ciência e Tecnologia de Alimentos) - Universidade Federal da Paraíba, João Pessoa, 2007. 
40 SANGSEETHONG, K.; LERTPHANICH, S.; SRIROTH, K. Physicochemical properties of oxidized cassava starch prepared under various alkalinity levels. Starch/Stärke, v. 61, p. 92-100, 2009.

41 SHIRAI, M.A.; HAAS, A.; FERREIRA, G.F.; MATSUGUMA, L.S.; FRANCO, C.M.L.; DEMIATE; I.M. Características físicoquímicas e utilização em alimentos de amidos modificados por tratamento oxidativo. Ciên. e Tecnol. Alim., v. 27, n. 2, p. 239-247, 2007.

42 SILVA, R.M.; FERREIRA, G.F.; SHIRAI, M.A.; HAAS, A.; SCHERER, M.L.; FRANCO, C.M.L.; DEMIATE; I.M. Características físico-químicas de amidos modificados com permanganato de potássio/ácido lático e hipoclorito de sódio/ácido lático. Ciên. e Tecnol. Alim., v. 28, n. 1, p. 66-77, 2008.

43 SINGH, G.D.; BAWA, A.S.; RIAR, C.S.; SAXENA, D.C. Influence of heat-moisture treatment and acid modifications on physicochemical, rheological, thermal and morphological characteristics of indian water chestnut (Trapa natans) starch and its application in biodegradable films. Starch/Stärke, v. 61, p. 503-513, 2009.

44 SINGH, J.; McCARTHY, O.J.; SINGH, H. Physicochemical and morphological characteristics of New Zealand Taewa (Maori potato) starches. Carboh. Pol., v. 64, p. 569-581, 2008.

45 SMITH, R. J. Characterization and analysis of starches. In: WHISTLER, R. L.; PASCHALL, E. F. Starch: Chem. and technol., v.1, p. 620-625, Nova York, 1967.

46 SONG, X.; ZHU, W.; LI, Z.; ZHU, J. Characteristics and application of octenyl succinic anhydride modified waxy corn starch in sausage. Starch/Stärke, v. 62, p. 629-636, 2010.

47 SPSS Inc. SPSS statistics base 19.0: user's guide. Chicago, 2010.

48 STAHL, J.A.; LOBATO, L.P.; BOCHI, V.C.; KUBOTA, E.H.; GUTHOSKI, L.C.; EMANUELLI, T. Physicochemical properties of pinhão (Araucaria angustifólia, Bert, O. Ktze) starch phosphates. LWT, v. 40, p. 1206-1214, 2007.

49 TAKIZAWA, F.F.; SILVA, G.O.; KONKEL, F.E.; DEMIATE, I.M. Characterization of tropical starches modified with potassium permanganate and lactic acid. Brazilian Arch. of Biol. and Technol., v. 47, p. 921-931, 2004.

50 THOMAS, D.J.; ATWELL, W. Starches: practical guides for the food industry. Minnesota: Eagan Press, 1999.94 p.

51 VILPOUX, O.F. Processos de produção de fécula de mandioca: comparação Brasil, Tailândia e China. In: CEREDA, M.P.; VILPOUX, O.F. Tecnologia, usos e potencialidades de tuberosas amiláceas latino americanas. São Paulo: Fundação Cargill, 2003. p. 143-175.

52 WÜRZBURG, O.B. Modified starches: properties and uses. Boca Raton: CRC Press, 1989.277 p.

53 YONEMOTO, P.G.; CALORI-DOMINGUES, M.A.; FRANCO, C.M.L. Efeito do tamanho dos grânulos nas características estruturais e físico-químicas do amido de trigo. Ciên. e Tecnol. de Alim., v. 27, n. 4, p. 761-771, 2007.

52 TRAN, T.; PIYACHOMKWAN, K.; SRIROTH, K. Gelatinization and thermal properties of modified Cassava starches. Starch/Stärke, v. 59, p. 46-55, 2007.

53 ZHANG, M.; CHEN, H.; ZHANG, Y. Physicochemical, thermal, and pasting properties of Chinese chestnut (Castanea mollissima BI.) starches as affected by different drying methods. Starch/Stärke, v. 63, p. 260-267, 2011. 